\title{
La dirección comercial en época de pandemia: el impacto del covid-19 en la gestión de ventas
}

\author{
Jorge Bullemore-Campbell ${ }^{1}$ y Eduard Cristóbal-Fransi ${ }^{2}$ \\ (1) Facultad de Economía y Negocios. Universidad del Desarrollo, Avenida la Plaza 680, Las Condes, Santiago, Chile. \\ (correo-e: j.bullemore@udd.cl). \\ (2) Departamento de Administración de Empresas. Universidad de Lleida. Campus de Cappont, C/ Jaume II, 73. 25001. \\ Lleida, España. (correo-e: eduard.cristobal@udl.cat)
}

Recibido Ago. 20, 2020; Aceptado Oct. 21, 2020; Versión final Nov. 17, 2020, Publicado Feb. 2021

\begin{abstract}
Resumen
Los objetivos de esta investigación son entender los efectos de la crisis producida por el coronavirus en la actividad comercial de empresas, conocer qué herramientas están usando las empresas para poder mitigar los impactos de la crisis, y entender los citados impactos con un efecto temporal. Se realiza una encuesta administrada a directivos de más de 75 empresas durante los meses de marzo, abril, mayo, junio y julio de 2020. Los resultados muestran que la mayor parte de las empresas ha estado monitoreando la actividad de venta y los resultados comerciales con medidas relacionadas con llamadas a clientes existentes, oportunidades de negocios creadas y cotizaciones enviadas. Se afianza la creencia que no antes de dos meses se podrá retomar progresivamente la normalidad. Se concluye que las pandemias generan caídas en la productividad agregada de las economías de los países, lo que a su vez impacta en una caída mayoritaria en la actividad comercial y en las ventas de las organizaciones.
\end{abstract}

Palabras clave: ventas; covid-19; coronavirus; productividad comercial

\section{Sales in a time of pandemic: impact of covid-19 on sales management}

\begin{abstract}
The objectives of this research study are to determine the effects of the coronavirus crisis on the commercial activity of companies to determine what tools companies are using to mitigate the impacts of the crisis and to better understand impacts with a temporary effect. A survey was administered to managers from more than 75 companies during the months of March, April, May, June, and July 2020. The results show that most companies monitor sales activity and commercial results by implementing measures related to calling clients, to business opportunities, and to sent quotes. There is a strengthened belief that not before two months normality can progressively resume. It is concluded that pandemics generate drops in aggregated productivity of country's economies that result in commercial activity and sales drops.
\end{abstract}

Keywords: sales; covid-19; coronavirus; sales productivity 


\section{INTRODUCCIÓN}

Desde finales del año 2019 el COVID-19, el nuevo coronavirus, se ha extendido por todo el mundo. El 11 de marzo de 2020 la Organización Mundial de la Salud declaró que el COVID-19 podía ser caracterizado como una pandemia. Para evitar su propagación, la mayoría de los países han implementado restricciones sin precedentes tales como el cierre de las fronteras nacionales, la limitación a las reuniones públicas y el cierre de universidades escuelas, tiendas y restaurantes. En algunos casos, las ciudades y regiones están siendo confinadas (Craven et al., 2020), por lo que se estiman graves consecuencias para las economías de los países, las empresas y los consumidores. Si bien muchos gobiernos tenían dificultades financieras antes del brote por coronavirus, la caída en los ingresos por impuestos amenaza gravemente la capacidad de los estados tanto para continuar respondiendo al virus como en su capacidad para permanecer solventes (McDonald y Larson, 2020). Evaluar y entender el impacto de la pandemia de COVID-19 en las ventas y en la actividad comercial de las empresas es esencial. A la fecha de elaboración de esta investigación, tan solo se contaba con un limitado número de estudios sobre los impactos del Covid-19 en las áreas comerciales, entre los que podemos destacar a Hartmann y Lussier (2020) y Rodriguez y Boyer (2020). La mayor parte de los estudios realizados en la actualidad se enfocaron en los efectos de las pandemias en sectores específicos de la economía, tales como el de la venta de vacunas (Gupta et al. 2012). Se ha desarrollado una visión de esta problemática realizando un estudio longitudinal encuestando a directivos de más de 75 empresas que operan en Chile, consecutivamente durante los meses de marzo, abril, mayo, junio y julio de 2020. La enfermedad por coronavirus 2019 (Covid-19) surgió en diciembre 2019 en la ciudad de Wuhan y se extendió rápidamente por toda China.

En los últimos siglos, se han reportado varios casos de pandemias de influenza en el mundo. Así, por ejemplo, podemos destacar las epidemias que tuvieron lugar en el siglo XVI en Inglaterra y la del siglo XVIII en USA (Ayora-Talavera, 1999). En los últimos 100 años, la llamada gripe española de 1918 ha sido considerada la más grave, causando más de 20 millones de muertes en todo el mundo (Ayora-Talavera, 1999). Después de la segunda guerra mundial se puede destacar la influenza asiática (H2N2) de 1957, o la influenza (H2N3) de 1968, que produjeron una mortandad de cerca de un millón de personas (Kilbourne, 2006). De acuerdo con el último informe disponible de la Organización Mundial de la Salud (OMS, 2020), toda la evidencia disponible para COVID-19 sugiere que el SARS-CoV-2 tiene un efecto zoonótico. Muchos investigadores han podido observar las características genómicas de SARS-CoV-2 y han encontrado que la evidencia no respalda que SARS-CoV-2 sea una construcción de laboratorio (Andersen et al., 2020). En relación a la evolución de casos, la OMS confirma los siguientes datos en su último reporte situacional del 30 de junio de 2020 (ver Tabla 1). Sin lugar a dudas esta pandemia tiene un carácter global, y para mediados del año 2020 se encuentra en plena etapa de desarrollo. Con los datos al cierre del mes de junio 2020, a nivel Global se cuentan más de diez millones de casos de Covid-19, con una tasa de mortalidad inferior al $5 \%$. Las regiones con el mayor número de casos eran Europa y las Américas, sumando prácticamente el $76 \%$ de los casos totales.

Tabla 1: Casos Covid 19 al 30 de junio de 2020 (Organización Mundial de la Salud, 2020)

\begin{tabular}{|l|l|l|}
\hline \multicolumn{1}{|c|}{ Región } & \multicolumn{1}{c|}{ Casos } & \multicolumn{1}{c|}{ Fallecidos } \\
\hline Global & 10.185 .374 & 503.862 \\
\hline Europea & 2.692 .086 & 197.254 \\
\hline Américas & 5.136 .705 & 247.129 \\
\hline Mediterráneo Oriental & 1.058 .055 & 24.423 \\
\hline Pacífico Oeste & 215.566 & 7.440 \\
\hline Sureste Asiático & 784.931 & 21.593 \\
\hline África & 297.290 & 6.010 \\
\hline
\end{tabular}

Tal como se mencionó anteriormente, históricamente la humanidad ha sufrido varias pandemias. Tan solo en el siglo XX, han tenido lugar en los años 1918, 1957 y 1968. No obstante, las mejoras en la atención médica y los avances de la tecnología pueden reducir sus impactos sanitarios y económicos (Martin et al., 2020). Una pandemia como lo es el Covid-19 afecta a la población de un país por el cierre de escuelas y el ausentismo profiláctico de los trabajadores, lo que genera una baja en la actividad de las empresas de cualquier dimensión. Así, por ejemplo, el brote de SARS en 2003 mostró que incluso una enfermedad con un impacto sanitario relativamente pequeño puede tener importantes efectos económicos. A nivel mundial, se cree que el SARS infectó a unas 8.000 personas, y generó 800 fallecimientos (Cooper y Coxe, 2005). El Banco Asiático de Desarrollo estimó que el impacto económico del SARS fue de alrededor de 18 mil millones de dólares en Asia oriental, alrededor del $0,6 \%$ del producto interno bruto (Fan, 2003). De acuerdo a la investigación de Smith, et al., (2009), solo en el Reino Unido una pandemia de influenza podría reducir el producto interno bruto entre un $0,5 \%$ y un $4,3 \%$.

Una pandemia como la gripe en sí no es necesariamente la mayor preocupación desde una perspectiva macroeconómica, pero la combinación del cierre de escuelas y el absentismo profiláctico produce efectos de 
caída del producto interno bruto de entre 1,14\% y un 1,42\% (Smith et al., 2011). Por lo tanto, es importante considerar el impacto epidemiológico del cierre de escuelas y el ausentismo profiláctico y tratar de lograr un equilibrio entre las políticas que afectan a estos y las duraciones de ausencia que son necesarias desde una perspectiva de salud pública sin imponer impactos económicos innecesarios. Esto plantea un problema particular para los gobiernos, que deben tomar un camino difícil entre el asesoramiento para "continuar como de costumbre" y las medidas de "distanciamiento social" que pueden ralentizar la tasa de crecimiento de una epidemia. Según describe la investigación de Keogh-Brown, et al., (2010) el brote de 2003 del síndrome respiratorio agudo severo (SARS) mostró que los brotes de enfermedades infecciosas pueden tener notables impactos macroeconómicos: pérdidas del PIB por la enfermedad de aproximadamente entre el $0,5 \%$ y el $2 \%$, pero el cierre de las escuelas y el absentismo profiláctico pueden más que triplicar estos efectos. Así también, podemos mencionar la reacción de los mercados financieros a la pandemia por Covid-19 descrito en el estudio de Baker et al. (2020). Sin lugar a dudas, una pandemia global como el Covid-19 puede llegar a generar impactos sobre las economías de los países, las empresas de todo tamaño, y las personas, generando un aumento de los niveles de la tasa de desempleo, y un cambio en las prioridades de gasto fiscal e inversión privada.

\section{FACTORES DE DESEMPEÑO COMERCIAL}

La productividad comercial de las organizaciones ha sido objeto de múltiples investigaciones (Bullemore y Cristóbal-Fransi, 2015; Córdoba-Vega y Naranjo-Valencia, 2017; Goad y Jaramillo, 2014, Muñoz M.A. 2019). El meta-análisis desarrollado por Verbeke et al. (2011) elabora un balance del campo contemporáneo y sintetiza la evidencia empírica del período 1982-2008 en 268 artículos científicos. A pesar del progreso significativo en la investigación académica, desafortunadamente, nuestra capacidad de predecir el rendimiento de ventas no es el óptimo (Evans et al., 2012). Las fuerzas de ventas son las responsables de implementar la estrategia de marketing y ventas, y consiste en una estructura organizacional con vendedores que tienen roles definidos, que realizan actividades de ventas para que la empresa pueda satisfacer las necesidades del cliente y así lograr sus metas financieras (Bullemore y Cristóbal-Fransi, 2018). La estructura de ventas, vendedores y actividades. son el resultado de un conjunto de decisiones básicas, procesos, sistemas, y programas llamados los impulsores de efectividad de la fuerza de ventas (Zoltners et al., 2008).

Anderson y Oliver (1987) conceptualizaron el desempeño de las ventas como la evaluación de vendedores basados en lo que producen (es decir, resultados de ventas) así como lo que hacen (es decir, comportamientos de ventas). Así, por ejemplo, podemos mencionar, las unidades vendidas, los ingresos generados, la participación de mercado, entre otros. Mientras que los comportamientos de ventas deben incluir habilidades de venta y actividades de venta como, por ejemplo, las llamadas de ventas, la gestión del tiempo, las visitas de ventas, etc. Independientemente de cómo se defina el rendimiento, los gerentes juegan un papel clave para garantizar que el vendedor cumpla con los objetivos de rendimiento (Bullemore y Cristobal-Fransi, 2015). De acuerdo a la investigación de Verbeke et al. (2011) el desempeño de las fuerzas de ventas depende de múltiples factores, tales como percepción de los roles de vendedores, la aptitud, el nivel de habilidades, la motivación, los factores personales, y la organización y el entorno. Aunque el estudio del desempeño del vendedor tradicionalmente se ha centrado en las actividades y las relaciones de vendedores con clientes, la investigación reciente propone que las relaciones y actividades intraorganizacionales de vendedores también desempeñan un papel vital en impulsar el desempeño de las ventas (Bolander et al., 2015).

En las últimas décadas, la aplicación de sistemas de relación con el cliente (CRM o Customer Relationship Management) ha experimentado un rápido crecimiento en empresas de todo el mundo. De acuerdo al estudio de Rodriguez y Honeycutt (2011) la utilización de sistemas CRM impacta positivamente en la efectividad y el rendimiento del vendedor. La gestión de la relación con el cliente (CRM) es esencial para el éxito de las empresas modernas (Zeng et al., 2003). De manera que la predicción de la propensión a ganar en el canal de ventas es fundamental para una gestión de ventas efectiva (Yan et al., 2015). Según plantean Cespedes y Marsh (2017) para mejorar los resultados de ventas y definir los Indicadores Claves de Ventas relevantes en un negocio (Key Performance Indicators), se debe deconstruir el embudo de ventas, en actividades como: 1) Prospección: Ilamadas en frío, correos electrónicos, Linkedln, etc.; 2) Calificación: conversaciones iniciales encaminadas a filtrar a los prospectos meramente interesados de los reales, y determinar oportunidades calificadas; 3) Oportunidades avanzadas: discusiones con oportunidades calificadas; 4) Cierre: pasos finales para negociar y cerrar un acuerdo; 5) Postventa: servicio, cumplimiento de pedidos, posible personalización y actividades de implementación.

Jordan y Vazzana (2011) plantean tres pilares para lograr el éxito en ventas: gestión, métricas y metodología. En ese sentido revelan que hay tres niveles de métricas de fuerza de ventas: los resultados comerciales, los objetivos de ventas y las actividades de ventas. Las actividades de ventas corresponden a tareas más tácticas y gestionables, tal como hacer llamadas de ventas o uso del CRM. A la fecha de la escritura de este artículo no se encontró literatura científica publicada sobre los impactos que tiene la pandemia por Covid-19 en la 
actividad comercial de las empresas, como tampoco en las ventas, ni las modalidades de trabajo con las que las organizaciones han enfrentado la pandemia. Tan solo algunos artículos de consultoras internacionales como Mckinsey \& Company (Gavin et al., 2020).

Sobre la base de esta revisión de la literatura, se formularon los siguientes objetivos de investigación: 1) Entender cómo ha afectado la crisis por el coronavirus en la actividad comercial y en las ventas de las empresas; 2) Determinar qué herramientas están usando las empresas para poder mitigar los impactos de la crisis; 3) Conocer estos impactos con un efecto temporal, para lo cual se tomarán muestras mensuales durante la pandemia.

\section{METODOLOGÍA}

Para determinar los impactos de la crisis por coronavirus en la actividad comercial y en las ventas en Chile se recopilaron datos durante un período de cinco meses consecutivos (marzo a julio de 2020) de directivos de empresas de tamaño mediano o grande, y diversos sectores industriales que operan en Chile. Se escogieron a empresas representativas de la actividad económica del país, y con una facturación anual mínima de USD 1.000 .000 de acuerdo a la calificación del Servicios de Impuestos Internos. La muestra de directivos se eligió básicamente por el conocimiento o experiencia en materias relacionadas con la dirección comercial y ventas, por ello se seleccionaron aquellos que al menos ostentaran el cargo de sub-director de área.

La metodología de investigación a través de encuestas se usa ampliamente en marketing, y es importante que se sigan pautas estrictas para garantizar que se obtengan conocimientos significativos (Hulland et al., 2018). Si se realizan correctamente, las encuestas en línea tienen ventajas significativas sobre otros formatos como son el mayor alcance, la flexibilidad, la rapidez o la conveniencia entre otros (Evans y Mathur, 2018). Durante los meses de marzo, abril, mayo, junio y julio se envió una encuesta a una base de 250 directivos de empresas que operan en Chile. Para cumplir con los objetivos trazados se realizó un cuestionario con las siguientes preguntas: ¿Cómo ha impactado la crisis por el coronavirus en la actividad comercial (nuevas oportunidades de negocios) de su empresa?; ¿Cómo ha impactado la crisis por el coronavirus en las ventas (en dinero) de su empresa?; ¿Qué está haciendo su empresa para sobrellevar el impacto del coronavirus?; ¿Por cuánto tiempo espera que su organización sea afectada por la crisis del coronavirus?; ¿Cuál será el gran desafío de su equipo de ventas como efecto del coronavirus?; ¿Cuáles son los Indicadores Clave de Desempeño (en inglés KPls, Key Performance Indicators) más relevantes para monitorear a su equipo comercial durante la crisis del coronavirus? Adicionalmente el cuestionario contenía preguntas de identificación básica tales como el nombre de la empresa, sector industrial, y datos de contacto. Por su grado de aceptación en la investigación en ciencias sociales en las primeras dos preguntas sobre el impacto de la crisis se usó una escala Likert de 5 puntos. Seguidamente se preguntaba por la duración de la crisis en la que había cuatro opciones. Finalmente, en el caso de las preguntas sobre la reacción a la crisis se utilizaron formularios con alternativas múltiples.

En el envío de marzo se obtuvo una tasa efectiva de respuesta del $30 \%$ con un total de 75 cuestionarios completos. Durante el mes de abril, se obtuvo una tasa efectiva del $31,2 \%$ con 78 respuestas completas, y en el mes de mayo se recogieron 80 respuestas completas con una tasa de respuesta del $32 \%$. En el mes de junio se lograron 63 respuestas, con una tasa del $25.2 \%$. Finalmente, el mes de Julio se obtuvieron 75 respuestas. El sector de actividad de las empresas encuestadas se presenta en la tabla 2.

Tabla 2: Sector industrial empresas encuestadas por mes

\begin{tabular}{|l|l|l|l|l|l|}
\hline \multicolumn{1}{|c|}{ Actividad } & Marzo-20 & Abril-20 & Mayo-20 & Junio-20 & Julio-20 \\
\hline Industrial & $26.5 \%$ & $25.6 \%$ & $23.8 \%$ & $26.9 \%$ & $26.5 \%$ \\
\hline Servicios & $19.1 \%$ & $17.9 \%$ & $17.5 \%$ & $22.2 \%$ & $19.1 \%$ \\
\hline Consumo & $11.8 \%$ & $12.8 \%$ & $13.8 \%$ & $14.2 \%$ & $11.8 \%$ \\
\hline Financiero & $11.8 \%$ & $10.3 \%$ & $10.0 \%$ & $14.2 \%$ & $11.8 \%$ \\
\hline Alimentos & $7.4 \%$ & $7.7 \%$ & $7.5 \%$ & $8.0 \%$ & $7.4 \%$ \\
\hline TI & $8.8 \%$ & $6.4 \%$ & $6.3 \%$ & $8.0 \%$ & $8.8 \%$ \\
\hline Insumos Médicos & $2.9 \%$ & $3.8 \%$ & $5.0 \%$ & $2.9 \%$ & $2.9 \%$ \\
\hline Educación & $1.5 \%$ & $2.6 \%$ & $3.8 \%$ & $1.1 \%$ & $1.5 \%$ \\
\hline Telcos & $1.5 \%$ & $2.6 \%$ & $3.8 \%$ & $1.1 \%$ & $1.5 \%$ \\
\hline No responde & $2.9 \%$ & $3.8 \%$ & $2.5 \%$ & $1.1 \%$ & $2.9 \%$ \\
\hline Turismo & $2.9 \%$ & $2.6 \%$ & $2.5 \%$ & $1.1 \%$ & $2.9 \%$ \\
\hline Energía & $1.5 \%$ & $2.6 \%$ & $2.5 \%$ & $1.1 \%$ & $1.5 \%$ \\
\hline Imprenta & $1.5 \%$ & $1.3 \%$ & $1.3 \%$ & $1.1 \%$ & $1.5 \%$ \\
\hline Total & $100.0 \%$ & $100.0 \%$ & $100.0 \%$ & $100.0 \%$ & $100.0 \%$ \\
\hline
\end{tabular}




\section{RESULTADOS}

En relación al impacto que ha generado la crisis en la actividad comercial de las empresas, se pueden visualizar los resultados en la tabla 3 . En los tres meses bajo observación, marzo, abril y mayo, se puede apreciar que la suma de las opciones "ha caído significativamente" y "ha caído moderadamente" se sitúan entre un $76 \%$ y un $85,9 \%$. Se puede observar que durante el mes de marzo -y primero de la crisis- las opciones "ha mejorado moderadamente" y "ha crecido significativamente" suman un 14,67\%. En cambio, en el mes de julio -tras cuatro meses de transcurrida la crisis- suman un 17,81\%. De acuerdo al análisis de comparación de medias por la prueba de $t$ de Student de los meses de marzo y mayo (Tabla 4), no existe evidencia de diferencias significativas durante el tiempo observado sobre el impacto en la empresa.

Tabla 3: Impacto de la crisis por el coronavirus en la actividad comercial (nuevas oportunidades de negocios) de la empresa

\begin{tabular}{|l|l|l|l|l|l|}
\hline \multicolumn{1}{|c|}{ Respuestas } & Marzo-20 & Abril-20 & Mayo-20 & Junio-20 & \multicolumn{1}{c|}{ Julio-20 } \\
\hline Ha caído significativamente & $48.00 \%$ & $53.85 \%$ & $53.75 \%$ & $44.44 \%$ & $36.99 \%$ \\
\hline Ha caído moderadamente & $32.00 \%$ & $32.05 \%$ & $31.25 \%$ & $39.68 \%$ & $39.73 \%$ \\
\hline No ha caído ni crecido & $5.33 \%$ & $5.13 \%$ & $6.25 \%$ & $1.59 \%$ & $5.48 \%$ \\
\hline Ha mejorado moderadamente & $6.67 \%$ & $7.69 \%$ & $6.25 \%$ & $9.52 \%$ & $13.70 \%$ \\
\hline Ha crecido significativamente & $8.00 \%$ & $1.28 \%$ & $2.50 \%$ & $4.76 \%$ & $4.11 \%$ \\
\hline Total & $100.00 \%$ & $100.00 \%$ & $100.00 \%$ & $100.00 \%$ & $100.00 \%$ \\
\hline
\end{tabular}

Tabla 4: Comparación de medias por prueba t de Student sobre el impacto de la crisis en la empresa

\begin{tabular}{|c|c|c|c|c|c|c|}
\hline \multicolumn{7}{|c|}{ Comparación de Medias } \\
\hline Estadística Descriptiva & & & & & & \\
\hline$V A R$ & $N$ & Media & Desv.Est. & Varianza & Mínimo & Máximo \\
\hline mar-20 & 75 & 1,94667 & 1,24002 & 1,53766 & 1 & 5 \\
\hline jul-20 & 80 & 1,725 & 1,00599 & 1,01203 & 1 & 5 \\
\hline \multicolumn{7}{|l|}{ Reporte de medias } \\
\hline$V A R$ & Media & $95 \% \quad L C L$ & $95 \%$ UCL & & & \\
\hline mar-20 & 1,94667 & 1,66136 & 2,23197 & & & \\
\hline jul-20 & 1,725 & 1,50113 & 1,94887 & & & \\
\hline Diferencia de medias (1-2) & 0,22167 & $-0,13565$ & 0,57898 & & & \\
\hline \multicolumn{7}{|l|}{ Prueba t suponiendo varianzas iguales } \\
\hline Diferencia de Medias Hipotetizada & 0 & & & & & \\
\hline Diferencia de medias & 0,22167 & & & & & \\
\hline Varianza Combinada & 1,26625 & & & & & \\
\hline Estadístico de la Prueba & 1,2256 & & & & & \\
\hline Grados de Libertad & 153 & & & & & \\
\hline \multicolumn{7}{|c|}{$\mathrm{H} 1:$ Mu1 - Mu2 $\neq 0$ / No es igual (dos colas) } \\
\hline t Valor Crítico (5\%) & 1,97559 & Valor $\mathrm{p}$ & 0,22223 & $\mathrm{H} 1(5 \%)$ & rechazado & \\
\hline \multicolumn{7}{|c|}{ H1: Mu1 - Mu2 < 0 / Menos que (cola izquierda) } \\
\hline t Valor Crítico (5\%) & $-1,65487$ & Valor $p$ & 0,88888 & $\mathrm{H} 1(5 \%)$ & rechazado & \\
\hline \multicolumn{7}{|c|}{ H1: Mu1 - Mu2 > 0 / Mas que (cola derecha) } \\
\hline t Valor Crítico (5\%) & 1,65487 & Valor $p$ & 0,11112 & $\mathrm{H} 1(5 \%)$ & rechazado & \\
\hline
\end{tabular}

Ante la pregunta sobre el impacto que ha generado la crisis en las ventas de las empresas. podemos visualizar los resultados en la tabla 5. En los cinco meses bajo observación marzo, abril, mayo, junio y julio se puede apreciar que la suma de las opciones "ha caído significativamente" y "ha caído moderadamente" es un $79.73 \%, 85.9 \%, 86 \%, 85.48 \%$ y $79.46 \%$ respectivamente. Se puede observar que durante el mes de marzo las opciones "ha mejorado moderadamente" y "ha crecido significativamente" suman un $10.82 \%$, en cambio en el mes de julio-tras cuatro meses de transcurrida la crisis- suman un $17.81 \%$. De acuerdo al análisis de comparación de medias por la prueba de t de Student de los meses de marzo y julio (Tabla 6) sobre el impacto en las ventas, no existe evidencia de diferencias significativas durante el tiempo observado. 
Tabla 5: Impacto de la crisis por el coronavirus en las ventas (en dinero) en la empresa

\begin{tabular}{|l|l|l|l|l|l|}
\hline \multicolumn{1}{|c|}{ Respuestas } & Marzo 20 & Abril-20 & Mayo-20 & Junio-20 & Julio-20 \\
\hline Han caído significativamente & $47.30 \%$ & $44.87 \%$ & $52.50 \%$ & $46.77 \%$ & $39.73 \%$ \\
\hline Han caído moderadamente & $32.42 \%$ & $41.03 \%$ & $32.50 \%$ & $38.71 \%$ & $39.73 \%$ \\
\hline No han caído ni crecido & $9.46 \%$ & $5.13 \%$ & $5.00 \%$ & $4.84 \%$ & $2.74 \%$ \\
\hline Han mejorado moderadamente & $5.41 \%$ & $6.41 \%$ & $8.75 \%$ & $6.45 \%$ & $15.07 \%$ \\
\hline Han crecido significativamente & $5.41 \%$ & $2.56 \%$ & $1.25 \%$ & $3.23 \%$ & $2.74 \%$ \\
\hline Total & $100 \%$ & $100 \%$ & $100 \%$ & $100 \%$ & $100 \%$ \\
\hline
\end{tabular}

Tabla 6: Comparación de medias por prueba t de Student sobre el impacto de la crisis en las ventas

\begin{tabular}{|c|c|c|c|c|c|c|}
\hline \multicolumn{7}{|c|}{ Comparación de Medias } \\
\hline Estadística Descriptiva & & & & & & \\
\hline VAR & $N$ & Media & Desv.Est. & Varianza & Mínimo & Máximo \\
\hline Columna A (1) & 74 & 1,89189 & 1,12952 & 1,27582 & 1 & 5 \\
\hline Columna B (2) & 80 & 1,7375 & 0,99038 & 0,98085 & 1 & 5 \\
\hline \multicolumn{7}{|l|}{ Reporte de medias } \\
\hline VAR & Media & $95 \% L C L$ & $95 \%$ UCL & & & \\
\hline Columna A (1) & 1,89189 & 1,6302 & 2,15358 & & & \\
\hline Columna B (2) & 1,7375 & 1,5171 & 1,9579 & & & \\
\hline Diferencia de medias (1-2) & 0,15439 & $-0,18322$ & 0,492 & & & \\
\hline \multicolumn{7}{|l|}{ Prueba t suponiendo varianzas iguales } \\
\hline Diferencia de Medias Hipotetizada & 0 & & & & & \\
\hline Diferencia de medias & 0,15439 & & & & & \\
\hline Varianza Combinada & 1,12252 & & & & & \\
\hline Estadístico de la Prueba & 0,9035 & & & & & \\
\hline Grados de Libertad & 152 & & & & & \\
\hline \multicolumn{7}{|c|}{$\mathrm{H} 1:$ Mu1 - Mu2 $\neq 0$ / No es igual (dos colas) } \\
\hline t Valor Crítico (5\%) & 1,97569 & Valor $\mathrm{p}$ & 0,36769 & $\mathrm{H} 1(5 \%)$ & rechazado & \\
\hline \multicolumn{7}{|c|}{ H1: Mu1 - Mu2 < 0 / Menos que (cola izquierda) } \\
\hline t Valor Crítico (5\%) & $-1,65494$ & Valor $p$ & 0,81616 & $\mathrm{H} 1(5 \%)$ & rechazado & \\
\hline \multicolumn{7}{|c|}{ H1: Mu1 - Mu2 > 0 / Mas que (cola derecha) } \\
\hline t Valor Crítico (5\%) & 1,65494 & Valor $p$ & 0,18384 & $\mathrm{H} 1(5 \%)$ & rechazado & \\
\hline
\end{tabular}

En la siguiente pregunta se consultaba sobre el tiempo esperado de duración de la pandemia, lo cual podría pre-disponer en las acciones comerciales que podrían realizar las empresas. En la observación realizada durante el mes de marzo, la opción de largo plazo -duración superior a los 2 meses fue ligeramente superior a la suma de opciones menores a dos meses, 50,67\% versus 49,33\%. En cambio, en la observación realizada en el mes de julio, la opción de largo plazo era ampliamente superior, un $67.12 \%$ versus un $32.88 \%$. Los resultados se pueden observar en la tabla 7.

Dada la disminución en la actividad comercial y de las ventas, además de un ajuste en las expectativas de duración de la crisis por Covid-19 se consultó sobre qué actividades habían implementado las organizaciones. Destaca el uso de plataformas virtuales para tener reuniones con clientes, prospectos, y reuniones internas, alcanzando prácticamente un $90 \%$. A su vez, se puede observar que las empresas han dado mayores flexibilidades de pago a sus clientes, pasando de un 29,33\% en marzo a un 56,25\% en Julio. Así también, se puede mencionar el ajuste mayoritario en las metas y objetivos de ventas, pasando de un 33,33\% en el mes de marzo a un 55\% en mayo. Por último, es relevante mencionar que una gran mayoría de compañías ha eliminado viajes y reuniones presenciales. Los resultados se pueden ver en la tabla 8.

Tabla 7: Tiempo esperado de afectación en la empresa por la crisis del coronavirus

\begin{tabular}{|l|l|l|l|l|l|}
\hline \multicolumn{1}{|c|}{ Respuestas } & Marzo-20 & Abril-20 & Mayo-20 & Junio-20 & Julio-20 \\
\hline Menos de 4 semanas & $6.67 \%$ & $0.00 \%$ & $2.50 \%$ & $1.61 \%$ & $0.00 \%$ \\
\hline Entre 4 y 6 semanas & $14.67 \%$ & $6.49 \%$ & $6.25 \%$ & $4.84 \%$ & $15.07 \%$ \\
\hline Entre 6 y 8 semanas & $28.00 \%$ & $14.10 \%$ & $16.25 \%$ & $14.52 \%$ & $17.81 \%$ \\
\hline Más de 2 meses & $50.67 \%$ & $79.49 \%$ & $75.00 \%$ & $79.03 \%$ & $67.12 \%$ \\
\hline
\end{tabular}


Por último, se ha realizado un estudio de cuáles son aquellos Indicadores Clave de Desempeño (Key Performance Indicators o KPIs) más relevantes que se están usando para monitorear al equipo comercial durante la crisis del coronavirus. Es importante mencionar que se dio la opción múltiple, pues las empresas pueden usar diversos indicadores para medir tanto la actividad como los resultados de la gestión comercial. Llama la atención que algunos indicadores han subido consistentemente en su aplicación, tales como "reuniones agendadas" y "reuniones realizadas", pasando $17,81 \%$ a un $28,77 \%$; y de un $28,77 \%$; a un $39,73 \%$ respectivamente. La cantidad de dinero de los cierres de ventas crece consecutivamente hasta llegar a un $69,86 \%$ siendo el indicado más relevante en el mes de julio. Otros indicadores, tales como "llamadas a clientes existentes", "llamadas a nuevos prospectos", "oportunidades de negocios creadas" y "número de cierres de ventas" no obstante, se obtienen resultados dispares. Los resultados se pueden observar en la tabla 9.

Tabla 8: Acciones de la empresa para sobrellevar el impacto del coronavirus

\begin{tabular}{|c|c|c|c|c|c|}
\hline Respuestas & Marzo-20 & Abril-20 & Mayo-20 & Junio-20 & Julio-20 \\
\hline $\begin{array}{l}\text { No estamos haciendo nada en particular. Mantenemos la forma de } \\
\text { trabajo }\end{array}$ & $1.33 \%$ & $1.28 \%$ & $0.00 \%$ & $0.00 \%$ & $1.37 \%$ \\
\hline $\begin{array}{l}\text { Usamos plataformas virtuales (Teams/Zoom/ Webex/ otras) para } \\
\text { tener reuniones con clientes y/o prospectos }\end{array}$ & $84.00 \%$ & $92.31 \%$ & $90.00 \%$ & $93.55 \%$ & $89.04 \%$ \\
\hline $\begin{array}{l}\text { Usamos plataformas virtuales (Teams/Zoom/ Webex/ otras) para } \\
\text { tener reuniones internas }\end{array}$ & $86.67 \%$ & $89.74 \%$ & $88.75 \%$ & $90.32 \%$ & $90.41 \%$ \\
\hline Estamos dando mayores flexibilidades de pago a nuestros clientes & $29.33 \%$ & $51.28 \%$ & $56.25 \%$ & $51.61 \%$ & $45.21 \%$ \\
\hline Hemos ajustado las metas y objetivos de ventas & $33.33 \%$ & $47.44 \%$ & $55.00 \%$ & $66.13 \%$ & $58.90 \%$ \\
\hline Hemos eliminado los viajes & $69.33 \%$ & $82.05 \%$ & $78.75 \%$ & $75.81 \%$ & $71.23 \%$ \\
\hline Hemos cancelado las reuniones presenciales & $89.33 \%$ & $91.03 \%$ & $85.00 \%$ & $80.65 \%$ & $78.08 \%$ \\
\hline
\end{tabular}

Tabla 9: Indicadores Clave de Desempeño (Key Performance Indicators) más relevantes para monitorear al equipo comercial durante la crisis del coronavirus

\begin{tabular}{|l|l|l|l|l|l|}
\hline \multicolumn{1}{|c|}{ Respuestas } & \multicolumn{1}{|c|}{ Marzo-20 } & \multicolumn{1}{c|}{ Abril-20 } & \multicolumn{1}{c|}{ Mayo-20 } & \multicolumn{1}{c|}{ Junio-20 } & Julio-20 \\
\hline Llamadas a clientes existentes & $49.32 \%$ & $49.35 \%$ & $50.63 \%$ & $37.70 \%$ & $43.84 \%$ \\
\hline Llamadas a nuevos prospectos & $31.51 \%$ & $42.86 \%$ & $35.44 \%$ & $32.79 \%$ & $43.84 \%$ \\
\hline Reuniones agendadas & $17.81 \%$ & $25.97 \%$ & $29.11 \%$ & $24.59 \%$ & $28.77 \%$ \\
\hline Reuniones realizadas & $28.77 \%$ & $38.96 \%$ & $44.30 \%$ & $37.70 \%$ & $39.73 \%$ \\
\hline Oportunidades de negocios creadas & $63.01 \%$ & $75.32 \%$ & $64.56 \%$ & $70.49 \%$ & $60.27 \%$ \\
\hline Propuestas o cotizaciones enviadas & $60.27 \%$ & $50.65 \%$ & $50.63 \%$ & $55.74 \%$ & $52.05 \%$ \\
\hline Número de cierres de ventas & $67.12 \%$ & $70.13 \%$ & $63.29 \%$ & $70.49 \%$ & $69.86 \%$ \\
\hline Monto (en dinero) de cierres de ventas & $54.79 \%$ & $58.44 \%$ & $67.09 \%$ & $63.93 \%$ & $65.75 \%$ \\
\hline
\end{tabular}

Los principales indicadores claves de desempeño que las empresas han usado durante la pandemia son el número de cierres de ventas, oportunidades de negocios creadas y el monto (en dinero) de cierres de ventas. Llama la atención que, si bien están en proporción minoritaria, las llamadas a nuevos prospectos, y reuniones (agendadas y realizadas) aumentaron su participación en más de 10 puntos porcentuales.

\section{CONCLUSIONES}

Sin lugar a dudas la crisis generada por Covid-19 ha propiciado un gran cambio en la manera como los consumidores y las empresas están llevando a cabo sus procesos de compras. De la revisión de la literatura podemos concluir que las pandemias generan caídas en la productividad agregada de las economías de los países, lo que a su vez impacta en una caída mayoritaria en la actividad comercial y en las ventas de las organizaciones.

La mayor parte de las empresas encuestadas ha estado monitoreando la actividad comercial con medidas relacionadas con llamadas a clientes existentes, oportunidades de negocios creadas y cotizaciones enviadas. A su vez, han medido las métricas de resultados tales como el número de cierres y facturación de ventas. Para ello es clave la utilización de herramientas como los sistemas de gestión de relaciones con clientes (o CRM, en inglés Customer Relationship Management). Ante estos hechos los directivos deben acelerar y mejorar sus procesos de gestión comercial vía teletrabajo y teleconferencias. Por lo que se sugiere capacitar y formar a la fuerza de ventas en el uso de plataformas de trabajo online, así como en el desarrollo de aquellas competencias que los vendedores requieran para mejorar las tasas de conversión de oportunidades a 
negocios, dentro de las que podemos mencionar técnicas de ventas consultivas virtuales, escucha activa, empatía, negociación y de cierre de negocios.

De acuerdo con la investigación llevada a cabo se afianza la creencia de que la crisis originada por el coronavirus tendrá una duración bastante larga, y que no antes de unos meses se considera poder retomar progresivamente a la normalidad. Se considera que, para finales de 2020 en Chile, país donde se desarrolló esta investigación, los casos activos de Covid-19 habrán disminuido a números mínimos a diferencia de los países del hemisferio norte, tales como los pertenecientes a la Unión Europea o los Estados Unidos, donde la pandemia presenta un marcado aumento de casos. Esta crisis podría ser un hito histórico que cambie la forma en que los seres humanos nos relacionemos, y hagamos negocios, por tanto, durante este proceso las empresas deben prepararse y mejorar constantemente para retornas a una nueva normalidad. Los directivos deben apostar por la digitalización de la empresa en general y resaltar la importancia de medir la actividad comercial y los resultados de los vendedores, así como apoyarse en áreas de Marketing para mejorar el proceso de prospección.

En relación a las limitaciones de esta investigación se puede mencionar que el hecho de tener en cuenta la perspectiva de una sola muestra puede producir sesgos. Además, se ha aplicado solo a los directivos de un solo país. Como futuras líneas de investigación se podrían contrastar estos resultados de manera complementaria con las percepciones de vendedores, clientes y/o proveedores. Además, se podría ampliar la muestra a directivos de empresas de otros países para analizar si el componente cultural puede afectar a los resultados. Asimismo, se puede realizar una investigación de carácter cualitativo con entrevistas en profundidad a los gerentes para verificar su percepción de los problemas relacionados con las ventas y la pandemia.

Por último, sugerimos que para futuras líneas de investigación se realice un análisis de ecuaciones estructurales con PLS-SEM para ver como son y qué peso tienen las relaciones entre las diferentes variables implicadas en la gestión comercial afectadas por el COVID. Así mismo, sugerimos analizar el efecto para las empresas de explorar nuevas oportunidades y de explotar mejor las oportunidades existentes y los procesos de innovación.

\section{AGRADECIMIENTOS}

Los autores agradecen al Ministerio de Economía, Industria y Competitividad del Gobierno de España por la concesión del proyecto TURCOLAB (ECO2017-88984-R). A su vez, a todas aquellas personas que cooperaron con sus respuestas en las encuestas realizadas.

\section{REFERENCIAS}

Andersen, K. G., Rambaut, A., y otros tres autores, The proximal origin of SARS-CoV-2. https://doi.org/10.1038/s41591020-0820-9. Nature Medicine, 26(4), 450-452 (2020).

Anderson, E. y Oliver, R.L. Perspectives on behavior-based versus outcome-based salesforce control systems, https://doi.org/10.1177/002224298705100407, Journal of Marketing, 51(4), 76-88 (1987).

Ayora-Talavera, G. Influenza: Historia de una enfermedad, Revista Biomédica, ISSN 2007-8447, 10(1), 57-61 (1999).

Baker, S. R., Bloom, N., y otros cuatro autores, The unprecedented stock market reaction to COVID-19, https://doi.org/10.1093/rapstu/raaa008, The Review of Asset Pricing Studies (2020).

Bolander, W., Satornino, C. B., Hughes, D. E., y Ferris, G. R., Social networks within sales organizations: Their development and importance for salesperson performance, https://doi.org/10.1509/jm.14.0444, Journal of Marketing, 79(6), 1-16 (2015).

Bullemore, J. y Cristóbal-Fransi, E., La gestión de los recursos humanos en las fuerzas de ventas, un estudio exploratorio a través del Método Delphi aplicado a las empresas peruanas, https://doi.org/10.22451/5817.ibj2018.vol2.1.11014, Iberoamerican Business Journal, 2(1), 18-43 (2018).

Bullemore J. y Cristóbal-Fransi, E. Análisis de los factores relevantes de la dirección de ventas, Alta Dirección, ISSN $0002-$ 6549, 50(285), 68-73 (2015).

Cespedes, F. V., y Marsh, R. Find the Right Metrics for Your Sales Team, Harvard Business Review (website) (2017).

Cooper, S., y D. Coxe, An Investor's Guide to Avian Flu, Montreal: BMO Nesbitt Burns Research (2005).

Córdoba-Vega, J. M., y Naranjo-Valencia, J. C. Incidencia de la inversión en innovación en las ventas de productos innovadores. Evidencia empírica en empresas manufactureras de Colombia, http://dx.doi.org/10.4067/S071807642017000200017, Información Tecnológica, 28(2), 153-166 (2017).

Craven, M., Liu, L., Mysore, M., y Wilson, M. COVID-19: Implications for business. McKinsey \& Company, Global Editorial Services (2020). 
Evans, R., y Mathur, A. The value of online surveys: A look back and a look ahead, https://doi.org/10.1108/IntR-03-20180089, Internet Research, 28(4), 854-887 (2018).

Evans, R., McFarland, R. G., Dietz, B., y Jaramillo, F., Advancing sales performance research: A focus on five underresearched topic areas, https://doi.org/10.2753/PSS0885-3134320108, Journal of Personal Selling \& Sales Management, 32(1), 89-105 (2012).

Fan, E. SARS: Economic Impact and Implications, ERD Policy Brief No. 15, Economics and Research Department, Asian Development Bank, Manila, Philippines (2003).

Gavin, R. Harrison, y otros tres autores, The B2B digital inflection point: How sales have changed during COVID-19 (2020).

Goad, E. A., y Jaramillo, F. The good, the bad and the effective: a meta-analytic examination of selling orientation and customer orientation on sales performance, https://doi.org/10.1080/08853134.2014.899471, Journal of Personal Selling \& Sales Management, 34(4), 285-301 (2014).

Gupta, V., Dawood, F. S., Muangchana, C.y otros trece autores. Influenza vaccination guidelines and vaccine sales in southeast Asia: 2008-2011, https://doi.org/10.1371/journal.pone.0052842, PloS one, 7(12), e52842 (2012).

Hartmann, N., y Lussier, B. Managing the sales force through the unexpected exogenous COVID-19 crisis, https://doi.org/10.1016/j.indmarman.2020.05.005, Industrial Marketing Management, 88 (2020).

Hulland, J., Baumgartner, H., y Smith, K. M., Marketing survey research best practices: evidence and recommendations from a review of JAMS articles, https://doi.org/10.1007/s11747-017-0532-y, Journal of the Academy of Marketing Science, 46(1), 92-108 (2018).

Jordan, J., y Vazzana, M., Cracking the sales management code: The secrets to measuring and managing sales performance, 1a edición, McGraw Hill Professional (2011).

Keogh-Brown, M. R., Smith, R. D., Edmunds, J. W., y Beutels, P. The macroeconomic impact of pandemic influenza: estimates from models of the United Kingdom, France, Belgium and The Netherlands, https://doi.org/10.1007/s10198-0090210-1, The European Journal of Health Economics, 11(6), 543-554 (2010).

Kilbourne, E. D. Influenza pandemics of the 20th century, https://doi.org/10.3201/eid1201.051254, Emerging Infectious Diseases, 12(1), 9-14 (2006)

Martin, A., Markhvida, M., Hallegatte, S., y Walsh, B. Socio-economic impacts of COVID-19 on household consumption and poverty. https://doi.org/10.1007/s41885-020-00070-3 Economics of Disasters and Climate Change, 4(3), 453-479 (2020).

McDonald, B. y Larson, S. Implications of the Coronavirus on Sales Tax Revenue and Local Government Fiscal Health, http://dx.doi.org/10.2139/ssrn.3571827 (2020).

Muñoz, M. Establecimiento de un Régimen de Facilitación del Comercio en Chile: Estudio del Comercio Internacional desde la Perspectiva de las Transiciones Socio-técnicas, http://dx.doi.org/10.4067/S0718-07642019000600233, Información Tecnológica, 30(6), 233-242 (2019).

Organización Mundial de la Salud, Coronavirus disease 2019 (COVID-19) Situation Report 101 (2020).

Rodriguez, M., y Honeycutt Jr, E. D. Customer relationship management (CRM)'s impact on B to B sales professionals' collaboration and sales performance, https://doi.org/10.1080/1051712X.2011.574252, Journal of Business-to-Business Marketing, 18(4), 335-356 (2011).

Rodriguez, M., y Boyer, S. The impact of mobile customer relationship management (mCRM) on sales collaboration and sales performance, https://doi.org/10.1057/s41270-020-00087-3, Journal of Marketing Analytics, 8(3), 137-148 (2020).

Smith, R. D., Keogh-Brown, M. R., Barnett, T., y Tait, J. The economy-wide impact of pandemic influenza on the UK: a computable general equilibrium modelling experiment, https://doi.org/10.1136/bmj.b4571, British Medical Journal, 339, b4571 (2009).

Smith, R. D., Keogh-Brown, M. R., y Barnett, T. Estimating the economic impact of pandemic influenza: an application of the computable general equilibrium model to the UK, https://doi.org/10.1016/j.socscimed.2011.05.025, Social Science \& Medicine, 73(2), 235-244 (2011).

Verbeke, W., Dietz, B., y Verwaal, E. Drivers of sales performance: a contemporary meta-analysis. Have salespeople become knowledge brokers? https://doi.org/10.1007/s11747-010-0211-8, Journal of the Academy of Marketing Science, 39(3), 407-428 (2011).

Yan, J., Zhang, C.y otros seis autores. On machine learning towards predictive sales pipeline analytics, In Twenty-ninth AAAI conference on artificial intelligence, AAAI Publications, Austin, USA (2015).

Zeng, Y. E., Wen, H. J., y Yen, D. C. Customer relationship management (CRM) in business-to-business (B2B) ecommerce, https://doi.org/10.1108/09685220310463722, Information Management \& Computer Security, 11(1), 39-44 (2003)

Zoltners, A. A., Sinha, P., y Lorimer, S. E., Sales force effectiveness: A framework for researchers and practitioners, https://doi.org/10.2753/PSS0885-3134280201, Journal of Personal Selling \& Sales Management, 28(2), 115-131 (2008). 
